\title{
Les injonctions paradoxales de la communication interne en période de tensions organisationnelles
}

Contradictory Injunctions and Internal Communication in Periods of Organizational Tension

\section{Laurent Morillon et Arlette Bouzon}

\section{(2) OpenEdition Journals}

Édition électronique

URL : http://journals.openedition.org/edc/1047

DOI : $10.4000 /$ edc. 1047

ISSN : 2101-0366

Éditeur

Université Lille-3

Édition imprimée

Date de publication : 1 décembre 2009

Pagination : 189-202

ISSN : $1270-6841$

Référence électronique

Laurent Morillon et Arlette Bouzon, « Les injonctions paradoxales de la communication interne en période de tensions organisationnelles », Études de communication [En ligne], 33 | 2009, mis en ligne le 01 décembre 2011, consulté le 20 avril 2019. URL : http://journals.openedition.org/edc/1047 ; DOI : 10.4000/edc. 1047

Ce document a été généré automatiquement le 20 avril 2019.

(c) Tous droits réservés 


\title{
Les injonctions paradoxales de la communication interne en période de tensions organisationnelles
}

\author{
Contradictory Injunctions and Internal Communication in Periods of \\ Organizational Tension
}

Laurent Morillon et Arlette Bouzon

1 Molex (délocalisation et fermeture de l'usine de Villemur sur Tarn, 283 licenciements), Caterpillar (600 licenciements sur les sites de Grenoble et Echirolles), Freescale (fermeture programmée d'ici fin 2011 de l'usine de Toulouse, 1050 licenciements), Continental (fermeture du site de Clairvoix, 1120 licenciements)..., nulle journée ne se passe sans l'annonce de licenciements, fermetures de sites, délocalisations. Dans ce contexte, les dirigeants se trouvent confrontés à une situation paradoxale : ils doivent maintenir le concours actif et la motivation d'un personnel considéré depuis les années 1990 comme «capital précieux» de l'entreprise (Weil, 1990), tout en appliquant des décisions stratégiques de plus en plus difficiles à justifier (certaines d'entre elles étant prises hors du territoire national pour des raisons financières indépendantes de la productivité des acteurs locaux concernés). Dans cette optique, le service de la communication interne, principal «représentant légal médiatique» (Sfez, 1997), doit contribuer à l'atteinte des objectifs économiques de l'organisation (Tixier, 1996) en suppléant aux phénomènes de distanciation des individus et des entités, en développant les relations entre les salariés, un sentiment d'appartenance et in fine l'investissement et la fidélité à l'organisation. Mais en période de tensions, comment ce service peut-il répondre à de telles demandes? Déjà, dans les années 1970, Dimitri Weiss constatait que «le traditionnel journal d'entreprise a peur du conflit, l'évite, le supprime, en faisant semblant qu'il n'existe pas (...) il s'avance jusqu'au seuil de l'information sur des situations porteuses de conflit qu'il n'ose cependant jamais, ou presque, franchir » (Weiss, 1977). Vingt années plus tard, Denis Sureau, alors directeur de la communication interne du groupe Suez, s'étonnait lui aussi : "n'est-il pas absurde que la communication interne se taise ou perde les pédales aussitôt qu'il commence à se passer quelque chose de réellement important? C'est pourtant 
la réalité (...) quand la communication officielle se fait entendre, elle est trop souvent peu crédible, louvoyant entre la langue de bois (parler pour ne pas dire) et la langue de coton (parler pour ne rien dire), la censure au nom de la confidentialité, les arguments de circonstance, les propos lénifiants. A ce jeu, elle perd rapidement de sa crédibilité »(Sureau, 1995).

Soumis à la censure (voire à l'autocensure), le service de communication interne n'est-il pas condamné à produire des supports qui idéalisent et enchantent l'entreprise, en complet décalage avec la situation vécue par les salariés? Qu'en est-il des pratiques en 2009, à une époque où l'incertitude s'accroît, où les pressions externes et internes se font plus fortes, où la précarité des acteurs grandit ? De façon plus générale, quelle est la place de la communication interne dans le travail d'organisation (ensemble des ajustements et des coordinations entrepris afin de rendre possible l'action collective) en période d'instabilités? Pour réfléchir à ces questions, nous nous proposons de réinterroger scientifiquement les résultats d'une étude menée fin 2002 au sein d'une organisation marchande engagée dans une restructuration et confrontée à un certain nombre de tensions entre la direction, la hiérarchie intermédiaire et les employés du siège social. Cette contribution tentera ainsi de comprendre les multiples facettes de l'incertitude existant dans l'activité d'un service de communication interne et de cerner les processus par lesquels les risques correspondants sont construits, appréhendés et intégrés par les acteurs concernés. Si l'étude d'un seul terrain ne saurait permettre une généralisation des conclusions obtenues, cette recherche nous donne toutefois l'occasion de questionner plus avant le statut de la communication interne.

Dans une première partie, nous nous intéresserons aux réactions des salariés français à la suite des changements organisationnels dans les grandes entreprises ainsi qu'aux missions des services de communication interne. Dans une deuxième partie, le terrain d'investigation, la méthodologie ainsi que les principaux résultats de la recherche menée seront présentés. Enfin, dans une dernière partie, nous considérerons les injonctions paradoxales qui influent sur les enjeux, statuts et pratiques de la communication interne en période de tensions organisationnelles.

\section{Des entreprises internationalisées entre tensions et rupture}

4 Mondialisation de l'économie, globalisation croissante des secteurs d'activités, crises financière et économique... l'environnement des entreprises internationalisées s'avère actuellement instable et incertain. Dans ce contexte, leurs dirigeants se révèlent particulièrement réactifs, orchestrant fusions et acquisitions, délocalisations, fermetures de sites, réorganisations structurelle et méthodologique, introduction des ERP, internationalisation de fournisseurs et de clientèles. Pour les salariés concernés, ces évolutions ne vont pas sans tensions (Morillon, Romane, 2008). Confrontés aux réorganisations imposées, aux chiffres de suppressions d'emplois annoncés, aux mutations de la relation d'emploi (flexibilité, employabilité...), nombre d'entre eux ont perdu leurs illusions et estiment que le contrat psychologique (Robinson, Kraatz, Rousseau, 1994) les liant à leur structure d'appartenance est rompu. Ils considèrent que leur contribution personnelle et collective (temps, énergie, savoir faire,...) est supérieure aux récompenses organisationnelles octroyées (salaire, formation, promotion, sécurité...). L'angoisse ressentie semble d'autant plus forte que les raisons des évolutions 
organisationnelles semblent souvent méconnues ou incomprises (Morillon, 2008). Leurs réactions peuvent alors prendre différentes formes: replis, corporatisme, scepticisme, résistance au changement, rumeurs, démissions, procès, grèves, séquestrations... voire suicides. Les salariés, plus diplômés, informés, cultivés et critiques (Sureau, 1995), n'hésitent plus, «à exprimer leurs craintes, parfois de manière médiatisée, affirmant ainsi leur droit à être informés, à comprendre et à être écoutés » (Morillon, Romane, 2008).

5 A titre d'illustration, les cas de Molex à Villemur sur Tarn et de Continental à Clairoix, issus de l'actualité récente, semblent symptomatiques de cette dégradation du climat social et de cette perte de confiance. Les 283 salariés du site haut-garonnais de Molex (une entreprise américaine de connectique automobile) ont appris en octobre 2008 la fermeture prochaine de leur usine. Les causes officielles invoquées par les dirigeants sont le manque de rentabilité et la crise économique. Les salariés, par l'entremise de leurs syndicats, affirment dans les médias que le site serait en fait délocalisé en Chine et aux Etats-Unis et qu'un stock de "sécurisation " aurait déjà été créé aux Pays-Bas. Ils ont engagé une action en référé devant le tribunal de grande instance pour obtenir la suspension du plan de sauvegarde de l'emploi, estimant que le comité d'entreprise «n'a pas été loyalement informé ». Ils ont par ailleurs séquestré pendant vingt-six heures deux de leurs dirigeants. Les employés de Continental Clairoix (dans l'Oise) sont quant à eux confrontés à l'annonce de la fermeture de leur usine au plus tard en mars 2010 avec le licenciement de plus de 1000 personnes. Pourtant, le fabricant allemand a publié des résultats positifs pour sa division pneumatiques en 2008 et ce site enregistrait 28 millions d'euros de bénéfices. En outre, peu de temps avant cette annonce, lors d'un comité d'entreprise exceptionnel, les représentants syndicaux avaient reçu l'assurance "qu'à ce jour aucune fermeture de Clairoix n'est prévue ». Deux ans auparavant, les salariés avaient accepté (par l'entremise de la CFTC, syndicat majoritaire et de la CGC) une hausse du temps de travail hebdomadaire à 40 heures (sans hausse de salaire) contre 130 embauches et la promesse du maintien de leur emploi au moins jusqu'en 2012.

6 Pour les salariés des autres sites de ces entreprises, ainsi que pour ceux des secteurs particulièrement touchés par la crise (automobile, immobilier, banque,...), le scepticisme, la perte de confiance, les décalages entre leurs représentations et celles de leurs dirigeants (Bouzon, 2003 ; Giroux, 2000) sont patents.

Dans de telles situations, certaines des missions traditionnelles de la communication interne peuvent devenir problématiques; rappelons en effet que les services de communication interne ont pour missions le développement d'un lien social et d'un sentiment d'appartenance, la diffusion et le partage d'une culture commune, l'implication morale des salariés (Detrie, Meslin-Broyez, 2001). En effet, ces missions soutiennent l'idée que, dans une économie de la connaissance, la participation de chacun à l'œuvre collective va au-delà de la simple réalisation de tâches distribuées, indépendantes les unes des autres. Elle vise à atteindre, à plusieurs, un but inaccessible à l'acteur individuel en raison de la répartition des savoirs. Elle implique une adhésion à l'objectif commun et peut donc difficilement être imposée sans un certain assentiment des personnes concernées. Pour atteindre ces objectifs, les services de communication interne utilisent notamment des moyens de communication qui mettent en récit l'entreprise (Almeida, 2001) tels que le journal interne, Intranet ou l'affichage ; les discours y étant, quant à eux, sélectionnés, contrôlés, organisés, orientés dans le temps de manière linéaire et conçus en fonction d'un locuteur idéal (Maingueneau, 1998). Mais dans un contexte difficile, face aux inquiétudes grandissantes des salariés et à un environnement de plus en plus 
incertain, ces missions traditionnellement dévolues aux services de communication interne sont amenées à évoluer. Qu'en est-il alors de leurs pratiques?

\section{La communication interne en période de tensions organisationnelles}

8 Les entreprises actuelles adoptent de nouvelles formes d'organisation (alliance, partenariat, fonctionnement en réseau, externalisation, essaimage...) qui, combinées aux formules précédentes (groupe, siège, succursale, filiale...), forment des organisations dites " élargies », qui se définissent par la force et l'intensité des liens qui les associent ainsi que par des stratégies de collaboration accrue. Nous nous proposons d'étudier les pratiques de communication interne dans une telle organisation marchande, en phase de restructuration organisationnelle. Au-delà de l'exemple susceptible d'illustrer la variété des situations existantes, notre propos vise à engager une réflexion sur les choix stratégiques effectués et sur les modes de management associés sur le plan communicationnel. Nous nous centrons en cela volontairement sur les activités du service de communication interne, à l'exclusion des relations syndicales qui constituent dans le cas présent une des prérogatives de la direction des ressources humaines.

9 Cette entreprise française de grande distribution spécialisée emploie quatorze mille personnes et compte cent trois magasins en France. Leader européen et troisième mondial dans sa spécialité, elle réalise un chiffre d'affaires de plus de deux milliards d'euros. En 1998, en contrepartie de la prise de contrôle d'une chaîne de distribution, cette entreprise cède cinquante cinq pour cent de son capital à un groupe britannique. Fin 2002, ce dernier en prend le contrôle total après avoir lancé une OPA. Le nouveau groupe réalise alors un chiffre d'affaires de onze milliards d'euros et compte soixante mille employés. A la suite de cette acquisition, les actionnaires du groupe britannique exigent plus de rentabilité de l'entreprise française. Sans licenciements massifs, le concept des magasins est redéfini, les gammes uniformisées, les prix baissés. Cette entreprise de tradition paternaliste se voit également appliquer un management plus directif et enregistre de nombreux changements organisationnels. Parmi ceux-ci, l'unification des services centraux - qui induit des mutations, de nouveaux rattachements, des recompositions d'équipes mais également un certain nombre de licenciements et de mise à la retraite anticipée - semble engendrer tensions, replis identitaires, stéréotypes (Iribarne, 1998), rumeurs au sein du siège du groupe.

Le service de la communication interne établi hiérarchiquement au niveau du "groupe » est composé de deux personnes. Rattaché à la direction générale et indépendant de la direction des ressources humaines (chargée de la gestion du personnel et des relations syndicales), il produit un journal interne bilingue destiné aux seuls membres de l'encadrement du groupe (cadres supérieurs en grande majorité), qui traite essentiellement de l'actualité internationale (ouvertures, nominations, projets...). Le service utilise également des courriels pour diffuser des informations plus ponctuelles. En pleine réorganisation du siège du groupe, le responsable de ce service s'interroge alors sur les préoccupations des collaborateurs du siège, leur état d'esprit, leurs avis sur les pratiques de communication interne et leurs attentes en matière d'informations. Il commande en septembre 2002 à un ancien collaborateur, devenu chercheur en sciences de l'information et de la communication, une étude en vue d'améliorer sa stratégie de 
communication. Pour ce faire, l'entretien individuel semi-directif est privilégié en raison de sa capacité à recueillir les opinions, croyances et/ou attitudes, comprendre les comportements et en analyser les causes profondes (Singly de, 2001). L'échantillon raisonné et diversifié composé de quinze personnes, respecte les principes d'hétérogénéité et de saturation. Les propos recueillis ont fait l'objet d'une analyse de contenu thématique dont une synthèse est présentée ici.

De façon relativement schématique, les entretiens font apparaitre que les réactions des salariés sont variables selon leur statut et leur histoire, leur attachement à l'entreprise, leur âge, leur précarité et leurs parcours académique et professionnel; elles oscillent entre repli identitaire, stéréotypes et amertume pour les uns, optimisme et opportunités négociées en interne ou en externe pour les autres. La première source de préoccupation concerne leur avenir professionnel et/ou une éventuelle prime de départ. Les incertitudes et les rumeurs correspondantes nourrissent méfiance et suspicion. Avec le départ des commandités, de plusieurs managers et la mutation du directeur des ressources humaines, certains salariés expriment un sentiment d'abandon. La confiance vis-à-vis des équipes anglaises est variable et leurs «faux pas» (ordres contradictoires, rattachements jugés incohérents...) difficilement pardonnés. Depuis le rachat et surtout depuis la prise de pouvoir des équipes anglaises, les acteurs interrogés estiment que l'ambiance au siège est pesante, la période de transition est jugée longue et désagréable. Les personnes qui se déclarent confiantes disent souffrir de la pression de «ceux qui n'y croient plus ». En matière de communication interne, les salariés interviewés s'affirment en distanciation vis-à-vis des supports traditionnels (tels que le journal) qui ont tendance à idéaliser l'entreprise. Ils attendent davantage de «proximité", d'«interactivité » et de " transparence » notamment par l'organisation de réunions et d'entretiens individuels. Si les informations diffusées dans les supports lors de l'OPA ont été appréciées de manière positive, les avis sont plus critiques concernant celles accessibles depuis cette période. Jugées insuffisantes et incomplètes, elles semblent favoriser les rumeurs et alimenter les doutes. Les informations attendues portent sur l'avenir des postes et les propositions susceptibles d'être faites tant pour le travail que pour les primes de départ. Elles sont

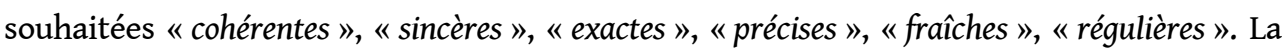
communication managériale apparaît dans ce contexte comme une opportunité pour le responsable de communication interne. Mais la hiérarchie intermédiaire officiellement chargée de cette forme de communication apparaît faiblement engagée tant par un défaut d'informations - d'autant que le degré d'exigence des salariés est élevé - que par les incertitudes quant à leur propre avenir (Palpacuer, Seignour, Vercher, 2007). Privés de ce levier d'action et dans un tel contexte de changement et de tensions, les objectifs fixés au service de communication interne générale du groupe sont-ils réalistes?

\section{Des injonctions paradoxales à l'autocensure}

Le point de vue fonctionnaliste de cette entreprise a induit une étude prescriptive, « appliquée » et utilitaire, dont l'objectif affiché a consisté à améliorer l'efficacité et/ou optimiser les actions du service communication. Constatant ses limites, nous avons essayé, dans le présent article, de dépasser cette visée en adoptant une posture scientifique. Ce choix a consisté à se détacher du modèle managérial au profit d'une démarche et d'une approche processuelles de l'analyse de l'organisation. Nous nous sommes inscrits dans la théorie de l'action interactionniste (Bernoux, 2004), qui a pour 
objet l'appréhension des transformations de l'organisation par la compréhension des comportements individuels. Ce changement de l'angle d'observation, qui fait rupture, nous a conduit à une redéfinition de l'organisation, devenue terrain d'observation du passage du «dire» au «faire» dans un savant tressage, et de la communication considérée désormais comme "organisante» (Giroux, 1994 ; Bouzon, 2006). Nous nous appuyons pour la présente réflexion sur le matériau recueilli lors de cette étude : sa portée critique, détachée de la logique de la commande, servant ainsi notre analyse sur les enjeux, statuts et pratiques de la communication interne générale en période de tensions organisationnelles.

Nous émettons l'hypothèse que le service de communication interne subit en période de tensions organisationnelles des injonctions paradoxales simultanées qui deviennent à terme une véritable double contrainte (Wittezaele, 2008). La première injonction est issue des missions officiellement assignées par les directions. En période de tensions organisationnelles, ce service a pour objectifs d'annoncer les changements, de répondre aux questions des salariés (Feldman, Spratt, 2000), d'apaiser les craintes, de faire partager une dynamique collective et de reconstruire l'identité culturelle et organisationnelle (Giroux, 2000). Une seconde injonction est particulièrement prégnante dans ces périodes où la censure se renforce : au nom de la confidentialité, certaines informations plus ou moins stratégiques ne doivent pas être diffusées. Au final, ces deux injonctions s'interdisent mutuellement: le service doit « dire » mais « ne peut dire ». Dans le même temps, il ne peut pas «ne pas dire " puisqu'il «dit» en continuant à produire des supports (dont le contenu est «enchanté » ou parcellaire). Dans la pratique, la fracture existant entre les attentes de la direction et celles des salariés met ce service en position délicate. Il est une interface entre un employeur intégré dans une logique économique (de l'efficacité, des revenus et/ou du bénéfice) et des employés qui, représentant la logique du vécu, aspirent à un mieux-être et à davantage d'équité (Lambert, 1979). En période de changement, il doit donc parvenir à concilier les objectifs fixés par des directions qui souhaitent la "paix sociale " (Morillon, Romane, 2008) avec les attentes et craintes des salariés, sans divulguer d'informations confidentielles ou stratégiques. Cette primauté du commanditaire financeur est intégrée par les acteurs concernés au point d'induire des pratiques d'autocensure. Ainsi, une observation ethnographique au sein d'une agence de communication chargée de réaliser le journal interne d'une entreprise publique en réorganisation a mis au jour l'autocensure pratiquée lors de sa conception par le journaliste d'entreprise, les personnes interviewées et leurs supérieurs hiérarchiques, les amenant à dénaturer les propos rapportés, et se faisant, altérant leur crédibilité et celle de la direction générale qui favorise et cautionne ces pratiques (Both, 2006). Alors qu' " en majorité les communicateurs internes estiment avoir accès aux sources nécessaires et parfois confidentielles pour faire leur travail et disposer d'une bonne marge de manœuvre dans le choix des sujets traités ", ils alternent «langue de bois » et «langue de coton» (Huyghe, 1991) dans leurs pratiques quotidiennes. Particulièrement en période de tensions organisationnelles, les employés peuvent avoir alors l'impression d'être les «objets » d'une démarche instrumentale, sentiment susceptible d'accentuer davantage encore une distanciation, une méfiance, l'individualisme, voire l'intention de quitter l'organisation, ou l'amenuisement de leur implication au travail (Morillon, Romane, 2008).

Si de telles injonctions paradoxales semblent alimentées par le contexte - elles deviennent de plus en plus prégnantes et paralysantes au fur et à mesure que se dégrade le climat social de l'entreprise - celles-ci pourraient avoir pour origine la représentation 
historiquement productive de la communication au sein des organisations (Giroux, 1994). En effet, cette dernière s'inscrit dans un processus de domination et d'influence, voire de manipulation (Floris, 1996) et d'acculturation des salariés; la satisfaction des besoins psychologiques et sociaux étant assimilable à un outil de contrôle et d'amélioration du rendement parmi d'autres (Giroux, 1994). De manière fonctionnaliste, elle est envisagée comme une opération à piloter pour résoudre des problèmes (Mucchielli, Guivarch, 1998). Cette acception gestionnaire et économique centrée sur la recherche de rentabilité implique la rationalisation des activités pour favoriser la maîtrise et l'efficacité de l'organisation. Le service de communication interne se trouve in fine, quel que soit son rattachement hiérarchique, perçu par les directions comme l'un de leur porte-parole et maintenu en conséquence dans un rôle de prestataire technique, sans pouvoir politique (Morillon, Romane, 2008).

\section{Pour conclure}

Restructurations, délocalisations, acquisitions... les occasions de tensions au sein des organisations se multiplient. Entre constat de complexité, injonctions et recettes, le sujet est aujourd'hui largement abordé tant dans les écrits professionnels que dans la littérature académique, en gestion et en sciences de l'information et de la communication. Mais trente années après les premiers travaux constatant les pratiques d'(auto)censure de la part des services de communication interne, ces derniers persistent souvent à jouer un rôle de "danseuse » ou de "magicien » de la direction (Corbalan, 1999) et ce malgré leur apparente professionnalisation. Si cette position apparaît contreproductive en période de tensions, elle semble toutefois relativement neutre une fois la stabilité retrouvée. Ainsi, dans l'entreprise considérée par exemple, en 2002 les salariés interviewés remettent en cause la crédibilité du journal et prennent leur distance vis-àvis de celui-ci lors de la réorganisation du siège. Pourtant, à peine deux ans plus tard, en 2004, une étude quantitative menée sur l'ensemble des salariés de l'enseigne française atteste d'une appréciation positive et même d'un fort attachement au journal national (Morillon, 2008) ! Les raisons profondes d'une telle évolution demeurent une énigme qui mériterait une nouvelle recherche.

Si nous avons privilégié dans notre propos la confrontation des résultats d'une enquête aux références susceptibles de nourrir notre réflexion sur le plan communicationnel, l'étude d'un seul terrain ne saurait cependant permettre une généralisation de nos conclusions.

La confrontation de nos travaux avec ceux notamment de B. Romane (thèse en cours) sur les changements dans le secteur des télécommunications confirme certains constats développés ici (Morillon, Romane, 2008). L'ensemble de ces réflexions sur le rôle de la communication interne incite alors à se questionner de manière plus large sur la place de l'individu dans l'organisation et sur l'exercice du pouvoir. 


\section{BIBLIOGRAPHIE}

Abdelmalki, L., Foulard, C. et al., (1998), L'entreprise communicante, Paris, Hermès

Almeida, N., (2001), Les Promesses de la communication, Paris, PUF.

Bernoux, P., (2004), Sociologie du changement dans les entreprises et les organisations, Paris, Ed. du Seuil.

Both, A., (2006), Le journal interne d'entreprise, Ethnographie d'une mission impossible?, in Ethnologie française, vol. $36, \mathrm{n}^{\circ} 1$, pp. 45-54, Paris, PUF.

Bouzon, A., (2003), Homogénéité des représentations dans l'entreprise : illusions et réalités, in Communication et organisation, $\mathrm{n}^{\circ} 22$, p.172-185.

Bouzon, A., (2006), Etudier la communication organisationnelle : champs, concepts, perspectives. Paris, L'Harmattan, collection Communication des organisations

Corbalan, J-A., (1999), Repenser la communication interne, in Communication et langages, $\mathrm{n}^{\circ} 120$, p.27-40.

Dejours, C., (1998), Souffrance en France, Paris, Ed. du Seuil.

Denoun, P., (1995), Identité, culture, entreprise, Paris, Publications de l'université Paris VII.

Detrie, P., Meslin-broyez, C., (2001), La Communication interne au service du management, Paris, Ed. Liaisons.

Dussauge, P., Ramanantsoa, B., (1984), Les Mutinationales : champions nationaux ou citoyens du monde, une question d'identité, in Revue Française de gestion, $\mathrm{n}^{\circ}$ 47-48, p.97-103.

Feldman, M., Spratt, M., (2000), Fusionner, agir vite pour réussir les transitions, Paris, Village Mondial.

Floris, B., (1996), Communication d'entreprise et pouvoir managérial, in Sciences de la Société, n³9, p.37-52.

Giroux, N., (1994), La communication interne : une définition en évolution, in Communication et Organisation, $\mathrm{n}^{\circ} 5$, p.17-47.

Giroux, N., (2000), La Communication dans la « réorganisation » des fusions-acquisitions, in Communication, Vol.19, n², Québec, Ed. Nota bene, p.11-44.

Huyghe, F.B., (1991), La langue de coton, Paris, Robert Laffont

Iribarne, P., (1998), Cultures et mondialisation : gérer par delà les frontières, Paris, Ed. du Seuil

Jodelet, D., (1989), Les représentations sociales. Paris, 1989.

Kaufmann, J-C., (2001), L'entretien compréhensif, Paris, Nathan Université.

Ladmiral, J.R., Lipianski, E.M., (2003), La communication interculturelle, Paris, Armand Colin.

Lambert, J., (1979), L'information ascendante dans les entreprises, Paris, EME.

Maingueneau, D., (1998), Analyser les textes en communication, Paris, Dunod. 
Morillon, L., (2008), Les salariés et l'information en période de réorganisation : des supports de communication interne en distanciation, in Forum Euro-méditerranéen, «Penser la Méditerranée », Axe Gérer la dimension médiatique de son organisation, juin 2008, Tanger, Maroc.

Morillon, L., (2005), Dans un contexte de fusion interculturelle, quelle évolution des marqueurs de culture dans le journal de communication interne?, in Colloque international « Culture des organisations et DISTIC », Université de Nice, Nice, p.79-84.

Morillon, L., Romane, B., (2008), Changement organisationnel et communication interne, du fantasme managérial au vécu salarial, in Rogojinaru A., Bouzon A. (dir.), Fondements de la communication des organisations : confrontations et dynamiques internationales, Bucarest, Editions Tritonic, p.157-174.

Moscovici, S., (1976), La psychanalyse, son image et son public, Paris, P.U.F.

Mucchielli, A., Guivarch, J., (1998), Nouvelles méthodes d'étude des communications, Paris, Armand Colin.

Palpacuer, F., Seignour, A., Vercher, C., (2007), Sorties de cadre(s), Paris, La Découverte.

Robinson, S.L., Kraatz, M.S., Rousseau, D.M., (1994), Changing obligations and the psychological contract: a longitudinal study, in Academy of Management Journal, 37(1), p.137-152.

Sfez, L., (1997), La communication, Paris, PUF.

Singly (de), F., (2001), L'enquête et ses méthodes : le questionnaire, Nathan Université, Paris.

Sureau, D., (1995), Communication interne : le choc salutaire de la crise, in Humanisme et entreprise, $\mathrm{n}^{\circ} 302, \mathrm{p} .57-64$.

Tixier, M., (1996), Les outils de mesure de la communication, in Communication et organisation, $\mathrm{n}^{\circ}$ 10, p.139-163.

Weil, P., (1990), Communication oblige!, Paris, Ed. d'Organisation.

Weiss, D., (1977), Réflexions sur la communication et l'entreprise, in Colloque du CERP, Liège.

Wittezaele, J.-J., (2008), La double contrainte. L'héritage des paradoxes de Bateson, Bruxelles, De Boeck.

\section{NOTES}

1. La firme globalisée tisse un vaste réseau de relations en menant des activités conçues, organisées et conduites à l'échelle mondiale (Dussauge, Ramanantsoa, 1984). Ces actions ont pour ambition un accroissement de la souplesse, de l'efficience et de la domination du marché (Abdelmalki, Foulard, 1998), la réalisation d'économies d'échelles et la rémunération des actionnaires.

2. Enterprise Resource Planning soit un progiciel de gestion intégré (PGI)

3. http://tempsreel.nouvelobs.com/actualites/social/20090505.0BS5710/

molex__laccord_repoussant_la_fermeture_de_4_mois_a_ete_.html - dernière consultation : 5 mai 2009.

4. http://www.rue89.com/tag/continental - dernière consultation : 5 mai 2009.

5. Correspondant à des images mentales construites sur leur univers social, les « représentations sociales » des individus (Moscovici, 1961) conditionneraient en partie leurs comportements (Jodelet, 1989). 
6. L'Offre Publique d'Achat est une proposition faite à des actionnaires par une entreprise ou un groupe d'investisseurs d'acheter des titres côtés en bourse dans l'optique d'une prise de contrôle d'une entreprise cible.

7. Il existe par ailleurs, un service de la communication au siège de la filiale française qui produit depuis plus de vingt ans un journal et une vidéo généralistes à destination de tous les salariés ainsi que des affiches, des événements, des cédéroms, un Intranet... pour des publics ciblés.

8. Le terme échantillon, plutôt quantitativiste, est toutefois mal adapté dans une approche qualitative (Kaufmann, 2001).

9. Trois employés, quatre agents de maîtrise, huit cadres - dix hommes et cinq femmes -âgés de 26 à 55 ans - avec une ancienneté de moins d'un an à plus de vingt-cinq ans - représentant l'ensemble des services du siège groupe en France.

10. Par exemple : «Les anglais ne veulent jamais entendre parler de plan social »

11. Entendue comme une «communication de proximité portée par chaque responsable d'équipe pour favoriser l'appropriation de la stratégie de l'entreprise par ses collaborateurs et subalternes et pour contribuer à sa mise en cuvre » in Les cahiers de la communication interne, Cahier $n^{\circ} 20$ "Le manager : maillon faible ou atout de la communication », mars 2007, AFCI, Association Française de Communication Interne.

12. Issue de la psychiatrie et des travaux de l'anthropologiste Gregory Bateson, une double contrainte désigne deux obligations qui se contrarient en s'interdisant mutuellement.

13. Non abordée dans l'étude commanditée à l'origine de cette recherche.

14. Trends-Tendances, Les paradoxes du journal d'entreprise, Roularta, Bruxelles, 6 avril 2006, p.62-63

15. Ce même journal a d'ailleurs été utilisé suite à l'acquisition pour renforcer l'unité culturelle de la nouvelle entité (Morillon, 2005)

\section{RÉSUMÉS}

Nulle journée ne se passe sans l'annonce de licenciements, de fermetures de sites, de délocalisations. Une étude menée au sein d'une entreprise en phase de réorganisation nous permet de constater la fracture entre les pratiques du service de communication interne et les attentes des salariés. Elle met en évidence les tensions entre acteurs collectifs et individu(s) dans la production de l'organisation, au sein de laquelle les rapports classiques, s'ils continuent à exister, sont accompagnés de reconfigurations majeures. Elle nous donne l'occasion de questionner scientifiquement les injonctions paradoxales que subissent les services de communication interne en période de tensions organisationnelles.

No day seems to pass without the announcement of redundancies, site closures and relocations. A study carried out within a company during a reorganization phase indicated the discrepancies between the practices of the internal communications service and the expectations of the employees. It revealed the tension between the corporation's spokespeople and the individuals involved in production. Standard internal reports, when they continued to be issued, had undergone major reconfigurations. We were also given the opportunity to question and to study the paradoxical orders received by the internal communications services during periods of organizational tension. 
INDEX

Keywords : crisis, organizational tensions, internal communication, double bind

Mots-clés : crise, tensions organisationnelles, communication interne, injonctions paradoxales

\section{AUTEURS}

LAURENT MORILLON

Laurent Morillon est maître de conférences en Sciences de l'Information et de la Communication au sein du LERASS (Laboratoire d'Etudes et de Recherche Appliquées en Sciences Sociales). Ses recherches portent sur la reconnaissance de l'individu au travail ainsi que sur les processus communicationnels à l'œuvre dans les organisations, notamment celles qui évoluent dans l'incertain.

\section{ARLETTE BOUZON}

Arlette Bouzon est professeur de Sciences de l'Information et de la Communication, enseigne actuellement à l'Université Paul Sabatier, Toulouse 3 (France). Elle est membre du LERASS (Laboratoire d'Etudes et de Recherche Appliquées en Sciences Sociales) et anime la section Organisational and Strategic Communication de l'ECREA (European Communication Research and Education Association). Ses travaux portent sur les processus communicationnels, la prise de décision dans l'incertain et l'intelligence collective dans les organisations élargies. 